\title{
Diferenciais de salários no Paraná: uma análise empírica a partir do Censo 2000
}

Marcos Aurélio Andrade Rocha* Maria de Fátima Sales de Souza Campos

\section{Introdução}

A importância do mercado de trabalho na alocação do produto nacional entre os agentes econômicos é sumária. A inserção no mercado de trabalho é objetivo da maioria dos jovens, e a garantia do emprego é uma preocupação de todos os empregados. Os jovens se preparam não só para entrar no mercado de trabalho, mas, também, para obter salários que permitam atingir o nível de bem-estar desejado. A literatura tem evidenciado que o mercado de trabalho, formal ou informal, distribui rendimentos de forma diferenciada entre os trabalhadores. No entanto, quais são os atributos dos trabalhadores que garantem a diferença salarial entre eles? Esta questão, via de regra, contempla respostas de pesquisadores de diversas áreas de estudo: historiadores, sociólogos, administradores, economistas inter alia todos eles terão uma resposta. Provavelmente, cada um deles terá uma resposta diferente (como é usual).

O objetivo deste texto é estudar a desigualdade de salários no Estado do Paraná usando os microdados do Censo Demográfico levantado pelo Instituto Brasileiro de Geografia e Estatística - (IBGE) - para o ano 2000. Este estudo estima equações de salários por gênero e por raça, totalizando quatro agrupamentos de indivíduos: homens, mulheres, brancos e negros/pardos. Esta divisão foi feita buscando-se facilitar a análise comparativa entre gênero e raça no mercado de trabalho paranaense.

Utilizou-se a metodologia de Heckman (1979) para estimar as equações por máxima verossimilhança, cujo procedimento estima uma equação de seleção (modelo probit), de onde se deriva um parâmetro que é usado para corrigir os vieses de seleção amostral da equação salarial, que surgem por motivos que serão detalhados adiante. Assim, o método deriva duas regressões: nas equações de seleção são mensurados os determinantes de inserção dos indivíduos no mercado de trabalho e, nas equações de salário são mensurados os determinantes dos diferenciais de rendimento dos trabalhadores. Subjacente na forma como foram distinguidas as equações, a hipótese deste trabalho é de que existem diferenças substanciais, tanto na distribuição de rendimentos entre homens, mulheres, brancos, negros e

\footnotetext{
* Mestrando em Desenvolvimento Econômico pela UFPR. Endereço eletrônico: sir.mrocha@gmail.com

** Doutora em Economia pelo PIMES/UFPE e professora adjunta do Departamento de Economia da UEL.
} 
pardos pelo mercado de trabalho, quanto existe diferenças na forma como estes grupos decidem sua oferta de trabalho.

Buscou-se mensurar os determinantes da propensão dos trabalhadores se dedicarem ao emprego. Segundo Heckman (1979), os determinantes da ocupação dos grupos estudados (em gênero e em raça) devem diferir, porque as perspectivas levadas em consideração por esses grupos são distintas. Por exemplo, as mulheres levam em consideração a maternidade no momento de ofertar horas de trabalho, um problema que não é necessário ser levado em consideração pelos homens. Os negros podem ser desmotivados a oferecer seu trabalho devido à expectativa de enfrentar discriminação no ambiente de trabalho. Aspectos como esses determinariam, portanto, uma diversidade na composição de oferta de trabalho entre esses grupos. O problema que este trabalho se propõe é verificar se estas diferenças ocorrem e em quanto elas podem ser mensuradas.

O artigo encontra-se distribuído em quatro seções, sendo a primeira esta introdução. $\mathrm{Na}$ seção seguinte apresenta-se a base de dados e as filtragens empregadas. Na terceira seção são apresentados e discutidos os resultados obtidos com as estimações. A última seção reúne as principais conclusões do estudo.

\section{Base de Dados}

A base de dados utilizada neste estudo é o Censo Demográfico conduzido pelo IBGE no ano de 2000 para o Estado do Paraná. A amostra se restringiu a indivíduos com idade entre 18 e 56 anos, pois neste intervalo, em geral, as decisões sobre o nível de escolaridade são menos interferidas pelo planejamento da fertilidade, especialmente para as mulheres. São incluídos na amostra apenas indivíduos que não estejam estudando, como faz Garen (1984).

A amostra foi limitada a trabalhadores dos setores urbanos. Foram excluídos empregadores, estagiários e indivíduos que trabalham para o consumo próprio. Adotou-se o procedimento utilizado por Sachsida et. al (2004), que consiste em excluir da amostra indivíduos que possuem um salário hora extremamente alto, o que poderia viesar a análise. Por isso, a amostra é composta apenas de indivíduos cujo salário horário semanal esteja no intervalo entre $R \$ 1,00$ e $R \$ 500,00$. Contudo, na análise como amostra censurada, foram incluídos os indivíduos que não estavam trabalhando durante a pesquisa do Censo (ou seja, com rendimento igual a zero), o que faz parte do procedimento de Heckman (1979). Os 
indivíduos ocupados na administração pública e no setor agrícola foram também excluídos da amostra, como feito por Soares e Gonzaga (1999).

\section{A equação de salários estimada}

A equação de salários estimada é:

$$
\begin{aligned}
& \ln w=\beta_{0}+\beta_{1} S+\beta_{2} S^{2}+\beta_{3} \operatorname{Exp}+\beta_{4} \operatorname{Exp}^{2}+\beta_{5} S x E X P++\beta_{6} V E+\beta_{7} O U V E+\beta_{8} A N D A+ \\
& \beta_{9} C u r+\beta_{10} \text { Lond }+\beta_{11} M a r+\beta_{12} \text { Formal }+\beta_{13} C S D O+\beta_{14} C O M P+ \\
& \beta_{15} R E S P+\varepsilon \\
& \quad \text { onde: } \\
& \ln w \text { é o logaritmo neperiano do salário semanal por hora; } \\
& \quad \beta_{0} \text { é o intercepto da equação; } \\
& \quad S \text { relaciona os anos de estudo do indivíduo; } \\
& \quad S^{2} \text { representa os anos de estudo elevado ao quadrado. Esta variável tem a finalidade }
\end{aligned}
$$
de captar os rendimentos decrescentes da escolaridade;

Exp descreve os anos de experiência do indivíduo, de acordo com a proxy clássica estabelecida por Mincer: Exp = Idade-Escolaridade -6 ;

$\operatorname{Exp}^{2}$ representa os anos de experiência elevados ao quadrado, uma proxy que tem a função de captar os rendimentos decrescentes da experiência;

SxEXP é uma variável de interação entre a escolaridade e a experiência;

$V E$ é uma dummy que assume o valor 1 para os indivíduos que não apresentam nenhuma dificuldade visual e 0 para aqueles que apresentam desde uma pequena dificuldade permanente até os totalmente incapazes de enxergar;

OUVE é uma dummy que assume o valor 1 para os indivíduos que não apresentam nenhum dificuldade de audição e 0 para aqueles que apresentam desde uma pequena dificuldade permanente até os incapazes de ouvir;

$A N D A$ é uma dummy que assume o valor 1 para os indivíduos que não apresentam nenhuma dificuldade em caminhar ou subir escadas e 0 para aqueles que apresentam desde alguma dificuldade permanente até total incapacidade de andar;

Cur, Lond e Mar são dummies para Curitiba, Londrina e Maringá respectivamente. Neste caso, as dummies mensuram o diferencial médio auferido pelos trabalhadores que fazem 
parte dessas cidades com relação aos demais municípios do Estado do Paraná ${ }^{15}$. O objetivo da inclusão dessas variáveis é captar a dimensão da heterogeneidade geográfica dessas regiões na atribuição dos salários de seus trabalhadores;

Formal é uma dummy que assume o valor 1 se o indivíduo trabalha com carteira assinada e 0 se trabalha informalmente;

$C S D O$ é uma dummy que assume valor 1 se o indivíduo é casado e 0 caso contrário;

1.1.1.1.1 COMP é uma dummy que assume o valor 1 se o individuo vive em companbia de cônjuge ou companheiro(a) e 0 caso contrário;

RESP é uma dummy que assume valor 1 se o trabalhador é chefe de família responsável pelo domicílio, e 0 em caso contrário;

$\varepsilon$ é um termo de perturbação estocástica.

As variáveis das categorias CSDO e COMP merecem maiores considerações. Enquanto a primeira inclui indivíduos que se encontram formalmente em situação conjugal, a segunda considera aqueles indivíduos que coabitavam ou tinham coabitado com cônjuge ou companheiro (a), no período da entrevista do Censo do IBGE. Portanto, a variável COMP diz mais respeito à convivência formal e informal com o parceiro do que a uma situação civil estabelecida de casamento, como estão caracterizados os indivíduos na variável CSDO.

Foram estimadas equações separadas para homens, mulheres, indivíduos brancos e indivíduos negros e pardos. Esta escolha do trabalho na seleção dos grupos para as estimações das equações de salário se deve, em primeiro lugar, às restrições que a metodologia de Heckman (1979) determina para a estimação de grupos diferentes de indivíduos, que, devido ao gênero ou raça da qual façam parte, têm salários de reserva distintos. Em segundo lugar, a distinção dos grupos em gênero e raça feita facilita a posterior análise comparativa dos determinantes de comportamento desses grupos, e dos resultados salariais que eles negociam no mercado de trabalho.

\section{Resultados e discussão: resultados das equações de seleção}

A estimação das equações de seleção inclui algumas variáveis adicionais que descrevem importantes fatores de decisão de oferta de trabalho dos indivíduos, como as variáveis "Recebimento de Pensão" e "Recebimento de Aluguel". Além destas, há as variáveis

\footnotetext{
15 Curitiba, Londrina e Maringá foram selecionadas por serem regiões metropolitanas representativas, de acordo
} com a categorização de regiões metropolitanas paranaenses listadas no Censo do IBGE em 2000. 
que descrevem recebimento de rendas exógenas ao mercado de trabalho, denominadas rendas I, II e III. A Renda I está relacionada a rendimentos de pensão alimentícia, mesada ou doação. A Renda II está relacionada ao recebimento de renda mínima, bolsa-escola e segurodesemprego. A Renda III constitui recebimento de rendimentos como abono de permanência em serviço, pensão paga por seguradora ou previdência privada, juros de aplicações financeiras etc. Estas variáveis procuram mensurar como a renda recebida de forma exógena ao mercado de trabalho influencia a decisão de inserção no mercado de trabalho. A tendência é de que recebimento de renda exógena aprecie o salário de reserva dos trabalhadores e, portanto, reduza sua propensão a ocupação no mercado de trabalho.

Os coeficientes das equações de seleção diferem sensivelmente entre os grupos. A Tabela 1 descreve os resultados para os homens e para as mulheres. O teste de razão de máxima verossimilhança para a estatística rho, que mensura a correlação entre a equação de seleção e a determinação do salário, mostra que existe o viés de seletividade da amostra no modelo para os dois grupos de indivíduos analisados. Em todos os casos estudados neste trabalho, rho se mostrou estatisticamente significante, resultado que comprova o viés de seleção da amostra e a necessidade do uso da correção de Heckman (1979). O teste do determinante global de significância do modelo de máxima verossimilhança também é positivo para todos os modelos estimados.

Os coeficientes relacionados à decisão de ocupação dos trabalhadores nas equações de seleção se comportaram da forma como é observado na literatura. A escolaridade se revelou positiva para homens e mulheres na determinação da propensão destes indivíduos em participar do mercado de trabalho (ou na avaliação positiva dos indivíduos com relação a seus salários-reserva, seguindo a formalização de Heckman), embora seja um determinante maior no caso dos homens. 
Tabela 1. Equação de seleção de Heckman, para homens e mulheres

\begin{tabular}{|c|c|c|c|c|}
\hline Variáveis & $\begin{array}{l}\text { Coeficientes } \\
\text { para Homens }\end{array}$ & $|\mathbf{P}|>z$ & $\begin{array}{c}\text { Coeficiente } \\
\text { para Mulheres }\end{array}$ & $|P|>z$ \\
\hline Escolaridade & 0,10395 & 0,00 & 0,09981 & 0,00 \\
\hline Casados & 0,16032 & 0,00 & $-0,04136$ & 0,05 \\
\hline Companhia/Cônjuge & 0,17945 & 0,00 & $-0,23701$ & 0,00 \\
\hline Chefe de Família & 0,52069 & 0,00 & 0,48905 & 0,00 \\
\hline Sem Def. Visual & 0,17571 & 0,00 & 0,10407 & 0,00 \\
\hline Sem Def. Auditiva & 0,13652 & 0,00 & 0,09686 & 0,06 \\
\hline Sem Dif. Caminhar & 0,50830 & 0,00 & 0,2815 & 0,00 \\
\hline Londrina & 0,08484 & 0,03 & 0,10209 & 0,00 \\
\hline Maringá & 0,01559 & 0,07 & 0,12330 & 0,00 \\
\hline Curitiba & 0,16873 & 0,00 & 0,18010 & 0,00 \\
\hline Exp & $-0,00246$ & 0,10 & $3,84 \mathrm{E}-06^{*}$ & 0,99 \\
\hline EscxExp & 0,00019 & 0,26 & $-0,00047$ & 0,00 \\
\hline Recebimento de Pensão & $-0,39503$ & 0,00 & $-0,12847$ & 0,00 \\
\hline Recebimento de Aluguel & $-0,20148$ & 0,00 & $-0,03735^{*}$ & 0,12 \\
\hline Receb. Renda I & $-0,31684$ & 0,00 & $-0,15680$ & 0,00 \\
\hline Receb. Renda II & $-0,47376$ & 0,00 & $-0,24372$ & 0,00 \\
\hline Receb. Renda III & $-0,39474$ & 0,00 & $-0,18419$ & 0,00 \\
\hline Constante & -1.28469 & 0,00 & $-1,34582$ & 0,00 \\
\hline \multirow[t]{3}{*}{ Teste Razão Max. } & $\mathrm{H}_{0}:$ rho $=0$ & & $\mathrm{H}_{0}:$ rho $=0$ & \\
\hline & $\operatorname{Chi}^{2}(1)=$ & & $\mathrm{Chi}^{2}(1)=1.654,99$ & \\
\hline & $\operatorname{Pr}>\mathrm{Chi}^{2}=$ & & $\operatorname{Pr}>\mathrm{Chi}^{2}=$ & \\
\hline Tamanho da Amostra & 18.614 & & 27.176 & \\
\hline
\end{tabular}

Fonte: Resultados da pesquisa.

*Coeficientes estatisticamente não-significantes a um nível de $10 \%$.

O estado civil apresenta sinais contrários e significantes na seleção. Para os homens, a condição de casado aumenta a propensão de inserção no mercado de trabalho; para as mulheres, a condição de casada é negativa. Este resultado provavelmente se justifica pela predominância dos homens no papel do sustento familiar, mesmo atualmente. O casamento determina positivamente a inserção dos indivíduos masculinos no mercado, porém age de forma negativa na inserção das mulheres no mercado de trabalho. Entre os fatores determinantes para esse comportamento distinto de homens e mulheres quanto à decisão de trabalhar e o casamento, pode-se destacar que, enquanto a maiorias dos homens se vêem na posição de provedores de família quando se casam, as mulheres nessa situação se tornam donas-de-casa e convivem com a expectativa de maternidade. A "figura" da mulher como 
dona-de-casa ainda persiste na sociedade e atua como fator negativo na propensão a procura por emprego das mulheres casadas. A variável que descreve a influência da existência de companhia/cônjuge para a ocupação dos indivíduos tem sinais semelhantes à variável de casamento - positiva para homens, negativa para mulheres. A diferença é que a propensão negativa da ocupação das mulheres é bem maior para esta variável do que para a variável que descreve a situação de casamento.

As variáveis que representam características pessoais da saúde dos indivíduos ausência de dificuldades visuais, auditivas e de caminhar - aparecem todas contribuindo positivamente para a probabilidade de inserção no mercado dos indivíduos, com exceção da ausência de deficiência auditiva para a mulher, que não resultou estatisticamente significante a um nível de 5\%. A ausência de dificuldades para caminhar aparece, dentre estas categorias, como a mais significativa na equação de seleção, tanto para homens quanto para as mulheres.

$\mathrm{Na}$ categoria dos determinantes regionais da seleção para os homens, a capital Curitiba aparece como região mais atrativa para a ocupação, seguida na ordem por Londrina e Maringá. No caso das mulheres, a capital é seguida em importância por Maringá e Londrina, nessa ordem.

Os resultados na equação de seleção para as variáveis que representam os rendimentos auferidos de forma exógena ao trabalho, tais como recebimentos de aluguéis, pensões etc, mostraram-se negativos e significantes na propensão dos indivíduos participarem do mercado de trabalho, como é esperado. A equação considera os recebimentos de aluguel, pensão, Renda I, Renda II e Renda III. A categoria Renda II aparece nos resultados como a mais representativa na depreciação da probabilidade de ocupação dos indivíduos, tanto para os homens como para as mulheres. É compreensível que os rendimentos relacionados com subsistência mínima e/ou temporária para os indivíduos mais necessitados, como os itens que compõem a Renda II, tenham um maior peso negativo na propensão de inserção no mercado de trabalho, porque atingem, na maioria, a população de baixa renda. Para estes indivíduos, o recebimento de uma renda regular, mesmo baixa, implica em considerável fator de afastamento da ocupação.

Por fim, é interessante notar que a condição de chefe de família aparece, para homens e mulheres, como o principal determinante de ocupação. A razão disso é bastante intuitiva: as tarefas e responsabilidades a que essa posição submete os indivíduos tornam premente a necessidade de empregar-se para prover o sustento dos familiares. É um fato atestado inclusive para as mulheres, como mostram os resultados. A chefia feminina do lar é 
um fenômeno cada vez mais comum, devido à substancial inserção da mulher em todos os setores da sociedade. Este fato confirma também a hipótese de que as mulheres desempenham hoje papéis que antes eram exclusivos dos homens.

Os dados da Tabela 2 mostram os resultados da equação de seleção para duas categorias: indivíduos brancos, de um lado, e negros e pardos, de outro. A variável escolaridade aparece significante para os dois grupos, embora seja um determinante de inserção no mercado de trabalho bem superior para os brancos. O estado civil casado aparece com sinal positivo para brancos, negros e pardos. Por outro lado, a situação de convivência com um parceiro ou cônjuge aparece negativo para os brancos, e não-significante para negros e pardos. A condição de chefe-de-família é um atributo positivo de inserção para os dois grupos (com propensão maior para os brancos).

As variáveis que descrevem os atributos pessoais dos indivíduos (ausência de deficiências visuais, auditivas e de locomoção física) aparecem com resultados similares para brancos e negros/pardos. Dentre estes atributos, o mais significativo para a ocupação é a ausência de dificuldades para caminhar. A variável que descreve a ausência de deficiências auditivas aparece não significante para os dois grupos.

Os determinantes regionais de inserção no mercado de trabalho seguem a linha discutida na comparação entre homens e mulheres, estabelecendo Curitiba no topo, seguida por Londrina e Maringá, nessa ordem. A diferença é que, para os indivíduos negros e pardos, o atributo regional desses três municípios com relação ao resto do Estado na propensão a ocupação no mercado de trabalho aparece bem mais significativa do que para os indivíduos brancos. 
Tabela 2. Equação de seleção de Heckman para brancos e negros/pardos

\begin{tabular}{|c|c|c|c|c|}
\hline Variáveis & $\begin{array}{l}\text { Coeficientes } \\
\text { para Brancos }\end{array}$ & $|P|>z$ & $\begin{array}{l}\text { Coeficientes para } \\
\text { Negros e Pardos }\end{array}$ & $\mathbf{P} \mid>z$ \\
\hline Escolaridade & 0,09340 & 0,00 & 0,05533 & 0,00 \\
\hline Casados & $0,02126^{*}$ & 0,27 & 0,05978 & 0,03 \\
\hline Companhia/Cônjuge & $-0,04040$ & 0,00 & $0,04474 *$ & 0,14 \\
\hline Chefe de Família & 1,14182 & 0,00 & 0,94914 & 0,00 \\
\hline Sem Def. Visual & 0,13805 & 0,00 & 0,16004 & 0,00 \\
\hline Sem Def. Auditiva & $0,03219 *$ & 0,44 & $0,11059 *$ & 0,11 \\
\hline Sem Dif. Caminhar & 0,35097 & 0,00 & 0,49350 & 0,00 \\
\hline Londrina & 0,08957 & 0,00 & 0,15195 & 0,00 \\
\hline Maringá & 0,08551 & 0,01 & 0,10004 & 0,04 \\
\hline Curitiba & 0,10612 & 0,00 & 0,36103 & 0,00 \\
\hline Exp & $-0,00704$ & 0,00 & $-0,01026$ & 0,00 \\
\hline EscxExp & $-0,00037$ & 0,00 & 0,00132 & 0,00 \\
\hline Recebimento de Pensão & $-0,32386$ & 0,00 & $-0,00053$ & 0,00 \\
\hline Recebimento de Aluguel & $-0,14289$ & 0,00 & $-0,01091 *$ & 0,35 \\
\hline Receb. Renda I & $-0,36053$ & 0,00 & $-0,00011$ & 0,00 \\
\hline Receb. Renda II & $-0,47165$ & 0,00 & $-0,02679$ & 0,00 \\
\hline Receb. Renda III & $-0,38663$ & 0,00 & $-0,02597$ & 0,00 \\
\hline Constante & $-1,24943$ & 0,00 & $-1,36018$ & 0,00 \\
\hline Teste Razão Max. & & & & \\
\hline \multirow[t]{3}{*}{ Verossim. } & \multicolumn{2}{|l|}{$\mathrm{H}_{0}: \mathrm{rho}=0$} & \multicolumn{2}{|l|}{$\mathrm{H}_{0}:$ rho $=0$} \\
\hline & \multicolumn{2}{|l|}{$\mathrm{Chi}^{2}(1)=1.342,50$} & \multicolumn{2}{|l|}{$\mathrm{Chi}^{2}(1)=1553,67$} \\
\hline & \multicolumn{2}{|l|}{$\operatorname{Pr}>\mathrm{Chi}^{2}=0,0000$} & \multicolumn{2}{|l|}{$\operatorname{Pr}>\mathrm{Chi}^{2}=0,0000$} \\
\hline Tamanho da Amostra & 33.911 & & 11.061 & \\
\hline
\end{tabular}

\footnotetext{
Fonte: Resultados da pesquisa.

*Coeficientes estatisticamente não-significantes a um nível de $10 \%$.
}

Esperava-se que a variável experiência e o termo de interação entre escolaridade e experiência atuassem como determinantes positivos da inserção no mercado de trabalho. Os resultados aqui, no entanto, contrariam essa expectativa: a variável aparece negativa para os brancos e negros/pardos, com exceção apenas ao termo de interação entre escolaridade e experiência, que resulta positivo para negros/pardos. 
O recebimento de rendas exógenas ao mercado de trabalho aparece como determinante negativo de ocupação, tanto para brancos como para negros e pardos. O recebimento da Renda II também se confirma, para os indivíduos comparados, como o principal fator depreciador da inserção no mercado de trabalho. Também, pode-se observar o resultado interessante de que, para os negros e pardos, as rendas exógenas ao mercado de trabalho aparecem como determinantes bem menos significantes de depreciação da tendência à ocupação do que para os brancos. Em especial, o recebimento de aluguel, pensão e Renda I aparecem com reduzida influência negativa.

Mais uma vez, a condição de chefe de família aparece como o determinante mais forte de ocupação, tanto para os brancos como para negros e pardos.

Tabela 3. Equações de rendimentos para homens e mulheres

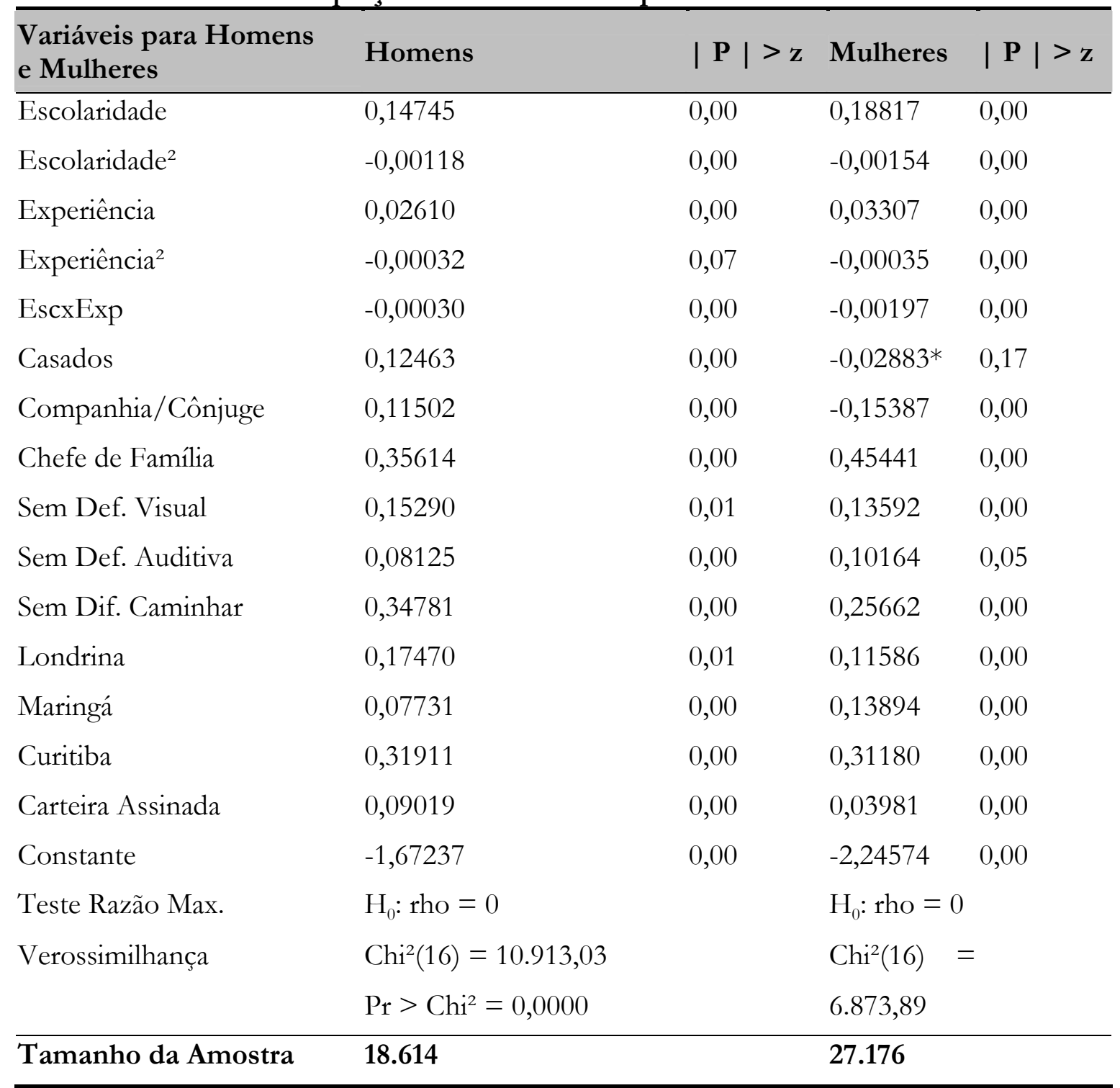

Fonte: Resultados da pesquisa.

*Coeficientes estatisticamente não-significantes a um nível de $10 \%$.

102 
Tabela 4. Equações de rendimentos para brancos e negros/pardos

\begin{tabular}{|c|c|c|c|c|}
\hline $\begin{array}{l}\text { Variáveis p/ Brancos e } \\
\text { Negros/Pardos }\end{array}$ & Brancos & $|\mathbf{P}|$ & Negros/Pardos & $|P|>z$ \\
\hline Escolaridade & 0,16418 & 0,00 & 0,04436 & 0.00 \\
\hline Escolaridade $^{2}$ & $-0,00104$ & 0,00 & $-0,00004$ & 0,00 \\
\hline Experiência & 0,02303 & 0,00 & $-0,00778$ & 0,00 \\
\hline Experiência $^{2}$ & $-0,00029$ & 0,00 & $-2,6 e-06$ & 0,00 \\
\hline EscxExp & $-0,00103$ & 0,00 & 0,00016 & 0,00 \\
\hline Casados & 0,06031 & 0,00 & 0,04688 & 0,03 \\
\hline Companhia/Cônjuge & 0,07447 & 0,00 & $0,03501 *$ & 0,14 \\
\hline Chefe de Família & 0,86872 & 0,00 & 0,74349 & 0,00 \\
\hline Sem Def. Visual & 0,16147 & 0,00 & 0,12539 & 0,00 \\
\hline Sem Def. Auditiva & $0,03330^{*}$ & 0,33 & 0,08656 & 0,07 \\
\hline Sem Dif. Caminhar & 0,28407 & 0,00 & 0,38647 & 0,00 \\
\hline Londrina & 0,14602 & 0,00 & 0,11912 & 0,00 \\
\hline Maringá & 0,11223 & 0,00 & 0,07832 & 0,04 \\
\hline Curitiba & 0,30854 & 0,00 & 0,28284 & 0,00 \\
\hline Carteira Assinada & 0,06329 & 0,00 & $0,000085^{*}$ & 0,14 \\
\hline Constante & $-2,02179$ & 0,00 & $-1,216927$ & 0,00 \\
\hline \multirow[t]{3}{*}{ Teste Razão Max. Verossimilhança } & \multicolumn{2}{|l|}{$\mathrm{H}_{0}:$ rho $=0$} & \multicolumn{2}{|l|}{$\mathrm{H}_{0}: \mathrm{rho}=0$} \\
\hline & \multicolumn{2}{|l|}{$\mathrm{Chi}^{2}(1)=1.342,50$} & \multicolumn{2}{|l|}{$\mathrm{Chi}^{2}(1)=4.868,15$} \\
\hline & \multicolumn{2}{|l|}{$\operatorname{Pr}>\mathrm{Chi}^{2}=0,0000$} & \multicolumn{2}{|l|}{$\operatorname{Pr}>\mathrm{Chi}^{2}=0,0000$} \\
\hline Tamanho da Amostra & 33.911 & & 11.061 & \\
\hline
\end{tabular}

Fonte: Resultados da pesquisa.

*Coeficientes estatisticamente não-significantes a um nível de $10 \%$.

A escolaridade aparece positiva e significante para todos os grupos analisados. As mulheres aparecem com o maior coeficiente para os retornos salariais à escolaridade: cada ano adicional na formação acadêmica lhes garante um acréscimo médio de 18\% em seus salários, enquanto os homens têm uma média de 14\%. O número que mais destoa do resultado geral é o coeficiente de escolaridade para negros e pardos, consideravelmente inferior aos demais. $\mathrm{O}$ modelo prevê um acréscimo salarial médio de apenas $4 \%$ a cada ano adicional de escolaridade para os negros/pardos, em contraste com os números para os grupos restantes. Esse resultado pode indicar severa discriminação do mercado de trabalho paranaense com relação 
aos indivíduos negros e pardos na hora de remunerar os indivíduos desta etnia com escolaridade idêntica aos outros grupos.

Os coeficientes da variável escolaridade ao quadrado aparecem negativos e significantes para a maioria dos grupos, confirmando a existência de rendimentos decrescentes para cada ano adicional de formação educacional.

A variável proxy experiência aparece positiva e significante para todos os grupos, indicando sua influência positiva da determinação dos rendimentos. A experiência ao quadrado resulta negativa e significante para todos os grupos de indivíduos analisados. Esses números confirmam o resultado apontado pela teoria do capital humano, que argumenta que um sinal da variável experiência ao quadrado negativa atesta rendimentos salariais decrescentes (embora positivos), para cada ano adicional de experiência do trabalhador.

Os indivíduos que estão casados ou vivem com companhia ou cônjuge recebem maiores salários; os resultados são bastante significativos, em especial para os homens. A exceção é dada pelos números estimados para as mulheres, que apresentam coeficientes não significantes para as casadas, e negativas para as que vivem com companheiros. Este resultado mostra uma espécie de "preferência", ou de discriminação do mercado de trabalho paranaense, em favor de mulheres que vivam independentes, sem companheiros. Esse fato pode derivar de considerações dos empregadores com relação à possível maior probabilidade de arcar com um período de maternidade para as mulheres que já vivem com seus parceiros. A variável companhia/cônjuge não aparece significante para negros e pardos.

A posição de chefe de família apresenta sinais positivos e significantes para os rendimentos de todos os grupos estudados. O grupo dos indivíduos brancos apresentou o maior coeficiente. É notável também que o coeficiente desta variável para as mulheres tenha se apresentado maior do que para os homens - reafirmando o fenômeno crescente da chefia feminina do lar e a valorização desta categoria pelo mercado de trabalho. As variáveis que relacionam atributos pessoais e/ou de saúde aparecem com resultados relativamente semelhantes para homens, mulheres, brancos e negros/pardos. A ausência de dificuldades para andar se estabelece para todos os grupos como o atributo mais importante na determinação dos rendimentos.

A influência regional nos salários mantém a capital Curitiba na dianteira dos resultados. Por sua vez, Londrina aparece na frente de Maringá na maioria dos resultados, embora Maringá ganhe a frente de Londrina na equação de rendimentos das mulheres. Os 
determinantes regionais aparecem mais modestos na equação dos negros e pardos, e mais significantes na equação para os brancos.

Por fim, cabe analisar uma variável específica da negociação no mercado de trabalho e que, por isso, não esteve presente nas equações de seleção: o atributo "Carteira Assinada", que distingue os trabalhadores do mercado formal dos trabalhadores informais. As equações mostram superioridade de salários para os trabalhadores com carteira assinada em todos os grupos analisados, exceto para negros e pardos, para os quais não aparece significante.

\section{Comentários Finais}

Este trabalho buscou estimar os determinantes dos rendimentos do trabalho no Estado do Paraná utilizando os microdados do Censo Demográfico do IBGE para o ano 2000. Os resultados obtidos confirmam o comportamento de muitas variáveis registradas na literatura: a escolaridade é um determinante positivo dos salários, assim como a experiência. Os retornos para a escolaridade dos indivíduos negros e pardos apresentaram-se bastante inferiores aos dos outros grupos, sinalizando uma possível discriminação salarial no mercado de trabalho paranaense. Os chefes de família têm um diferencial positivo de salário em relação aos indivíduos que se autodeclararam dependentes ou solteiros. Fazer parte do mercado de trabalho formal com carteira de trabalho assinada é um fator de aumento do nível médio de salários com relação ao mercado informal. $\mathrm{Na}$ análise regional, moradores da capital Curitiba levam grande vantagem na determinação de seus rendimentos em relação ao resto do Estado, sendo seguidos por Londrina e Maringá. Este fato pode-se dar devido à concentração populacional e econômica da região metropolitana de Curitiba, o que garantiria maior remuneração aos seus trabalhadores.

\section{Referências bibliográficas}

BECKER, G. Investment in Human Capital: A Theoretical Analysis. Journal of Political Economy, v.70, 1962.

EHRENBERG, R., SMITH, R. A Moderna Economia do Trabalho: Teoria e Política

Pública, Quinta Edição, Makron Books, 2000.

GAREN, J. The Returns to Schooling: A Selectivity Bias Approach with a Continuous Choice Variable. Econometrica, v. 52, n5, 1984.

HECKMAN, J. Shadow Prices, Market Wages, and Labor Supply. Econometrica, v. 42, no 4, 1974. . Sample Selection Bias as a Specification Error. Econometrica, v. 47, 1979. 
KASSOUF, A. L. Wage gender discrimination and segmentation in the brazilian labor market. Economia Aplicada, vol. 2, n 2, 1998.

MINCER, J. Schooling, Experience and Earnings. Columbia University Press: New York, 1974.

SACHSIDA, A., LOUREIRO, P., MENDONÇA, M. Os Retornos para a Escolaridade: Uma Abordagem do Viés de Seletividade com Escolha de Variável Contínua para o Brasil. Estudos Econômicos, vol. 58, nº2, 2004.

SOARES R., GONZAGA, G. Determinação de salários no Brasil: Dualidade ou não linearidade no retorno à educação. Revista de Econometria, vol. 19, nº 2, 1999. 